\title{
The Damper Performance Tests for the Aeolian Vibration of Overhead Lines
}

\author{
Wenping Xie, Tian Peng, Kai Xiao, and Jian Zhang
}

\begin{abstract}
According to Tongtangjia $220 \mathrm{kV}$ transmission line in Jiangmen, a city in southern China and IEC standard, Aeolian vibration experiments were designed and conducted in this paper. The tests investigated the an-vibration properties of stock-bridge dampers at different tension, simultaneous determinate the natural vibration frequencies of lines with or without dampers which, were compared with the theoretical calculating results and the numerical results. It shows that performances of dampers on the lines meet the standard of IEC. Besides, the frequencies of lines are in accordance with frequencies in theory and the calculating results of MATLAB. By comparison, MATLAB natural frequencies of conductor without damper are close to the theoretical method, and the frequencies would decrease with the addition of damper compared with the bare line. The no-dimensional damping coefficients at different tension should taken into consideration in anti-vibration design. And IEE standard is a good way to verity the damper performance.
\end{abstract}

Index Terms-Transmission lines, Aeolian vibration, stock-bridge dampers, laboratory test.

\section{INTRODUCTION}

Aeolian vibration known as wind-induced vibration, has a high frequency and small-amplitude motion, which arises from alternating forces caused by vortex shedding. The frequency of Aeolian vibration ranges from 3 to $50 \mathrm{~Hz}$ and the wind speed ranges from 1 to $7 \mathrm{~m} / \mathrm{s}$ [1]-[3].The amplitude of Aeolian vibration less than diameter of the conductor, which ranges from 6 to $50 \mathrm{~mm}$. Hence the vibration of the conductor produces altering bending stress and tensile stresses in the vicinity of the clamps. Eventually, this leads to fatigue damage of the conductor in the form of broken strands in the outer layer, usually at the point of contact such as the suspension clamp. Also, there are other damage types including the slippage of the messenger wire of the Stockbridge damper, insulator strings, and the loosening of parts. Now there are two methods to analyze the response of Aeolian vibration, the energy balance method and the dynamical method. But those methods cannot settle down the anti-vibration design of conductors due to the character, small amplitude, fluid-solid coupling, non-linearity of

Manuscript received July 13, 2016; revised October 23, 2016. This work was supported in part by Electric Power Research Institute of Guangdong Power Grid Co., Ltd, Guangzhou, 510080, China, and Tongji University, Shanghai, 200092, China..

W Xie is with Guangdong Electric Power Company Research Institute, Guangzhou, 510030, China (e-mail: 309577110@qq.com).

T. Peng is with the Department of Civil Engineering, Tongji University, Shanghai, 200092, China (e-mail:pt0796@163.com).

K. Xiao was with the Department of Materials Science and Engineering, Zhejiang University, Hangzhou, 310000 , China. He is with Guangdong Electric Power Company Research Institute, Guangzhou, 510030, China (e-mail: 13928750816@163.com). conductor and messenger of damper of Aeolian vibration. Now Aeolian vibration experiments are mainly applied to test the damper performance. Therefore, according to Tongtangjia $220 \mathrm{kV}$ transmission line in Jiangmen and the specification of IEC [4]-[6], Aeolian vibration experiments were designed and conducted to test the anti-vibration property of dampers in this paper.

\section{TEST APPARATUS}

The test was carried out in Shanghai Electric Cable Research Institute. The type of tested conductor are LGJ-500/45, with the $56 \mathrm{~m}$ span length and the $48.2 \mathrm{~m}$ free-span length. The whole arrangement shown in Fig. 1.

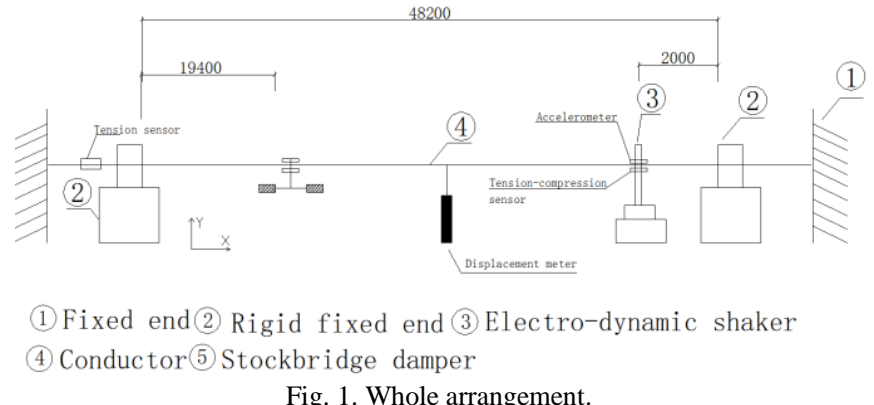

The conductor were tested at $20 \%$ and $30 \%$ RTS(rated tensile strength).The main apparatus contained fixed suspension system, tension system, excitation system, testing and data collecting system[7]. Excitation system utilized ESD-045 electro-dynamic shaker, which is a vibration exciter capable of generating sinusoidal force. The experimental analysis used the forced respond method. Measurements were taken the force transmitted by the shaker to the conductor, the acceleration of the conductor, and the phase angle between the force and the acceleration. The phase angle between the force and the acceleration reached $90^{\circ}$, a stable degree, through oscilloscope control, as shown in Fig.2, Fig. 3.

\section{EXPERIMENT RESULT ANALYSIS}

\section{A. Natural Frequency Analysis of Conductor without Damper}

The conductor natural frequency were analyzed by the time-history of the displacement of maximum amplitude point(Fig. 4) after stopped the stable external excitation vibration and used self-damper of conductor to eliminate vibration. The results are show in Fig. 5.

Equation (1)[8] is the theoretical natural frequency of 
the conductor without a damper.
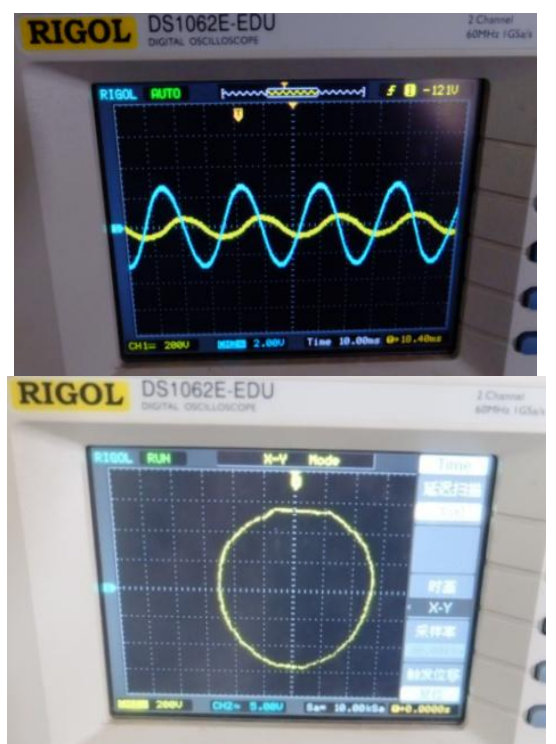

Fig. 2. Signals of force and acceleration.

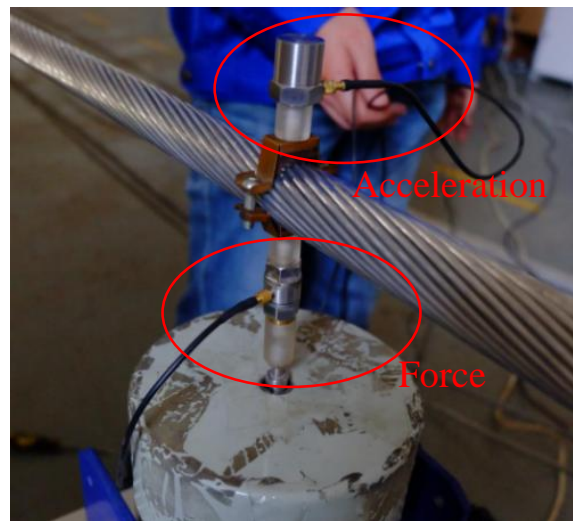

Fig. 3. Electro-dynamic shaker

$$
w_{n}=\frac{n \pi}{l} \sqrt{\frac{T_{0}}{m}} \sqrt{1+\frac{E J}{T_{0}}\left(\frac{\mathrm{n} \pi}{l}\right)^{2}}
$$

where $l$ is the length of conductor, $T_{0}$ is the tension of the conductor, $m$ is mass per unit length, $E J$ is the stiffness of the conductor.

MATLAB codes were made up to calculate the frequency of conductor with or without damper according to the finite element modeling proposed by Oumar Barry[9-11] based on the Hamilton's principle. Table I compares the natural frequency of the three methods of the conductor without damper.

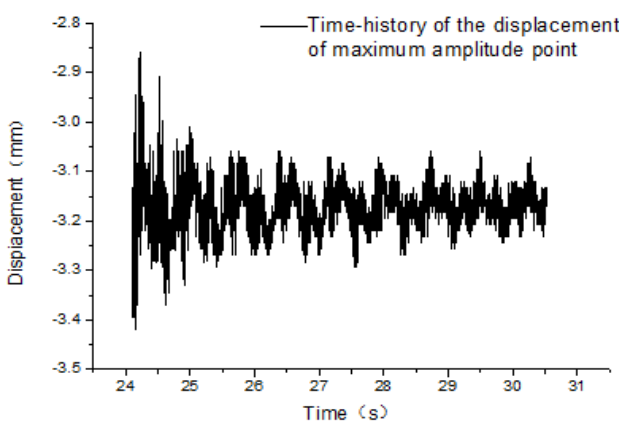

Fig. 4. Time-history of the displacement.

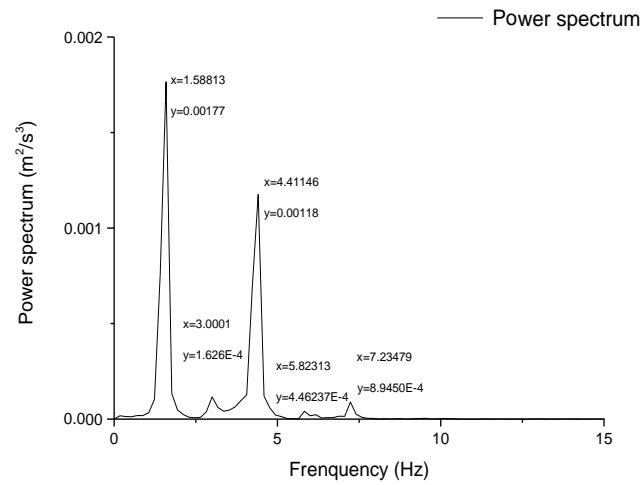

Fig. 5. Power spectrum

TABLE I: COMPARISON OF MATLAB, EXPERIMENTAL AND THEORETICAL

\begin{tabular}{llll}
\multicolumn{4}{c}{ NATURAL FREQUENCIES OF THE CONDUCTOR WITHOUT DAMPER $(\mathrm{Hz})$} \\
\hline Mode & Experimental & Theoretical & MATLAB \\
\hline 1 & 1.59 & 1.30 & 1.34 \\
2 & 3.01 & 2.60 & 2.62 \\
3 & 4.41 & 3.90 & 3.92 \\
4 & 5.82 & 5.20 & 5.23 \\
5 & 7.23 & 6.50 & 6.53 \\
\hline
\end{tabular}

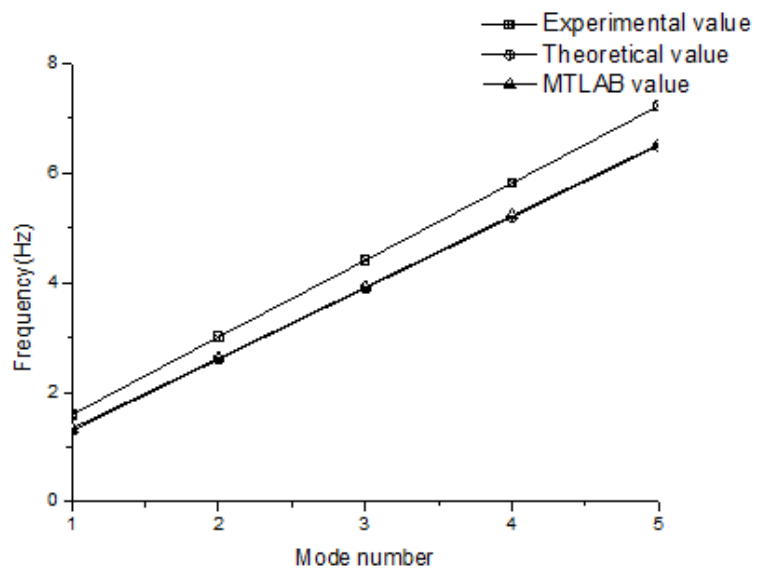

Fig. 6. Comparison of three methods of natural frequency

From Table I and Fig. 6, it is evident that three results are very close. As it can be seen, the natural frequencies found from the MATLAB method are identical to those from the theoretical equation.

\section{B. Natural Frequency Analysis of Conductor with Damper}

The natural frequency of conductor with damper differ in the different installation positions and type of damper, and now there is no general formulas to calculate the dynamic property of conductor with damper. Table II compares the natural frequency of experimental and MATLAB value.

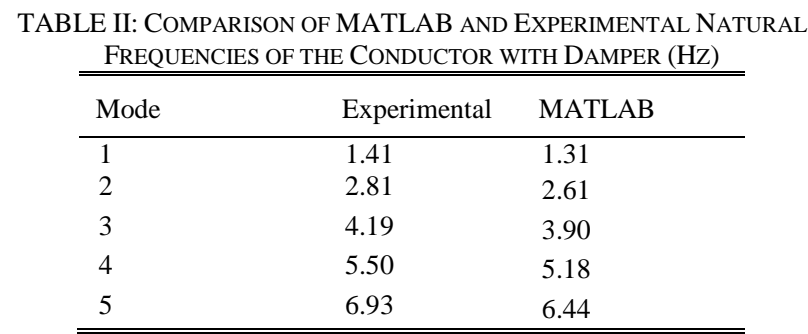




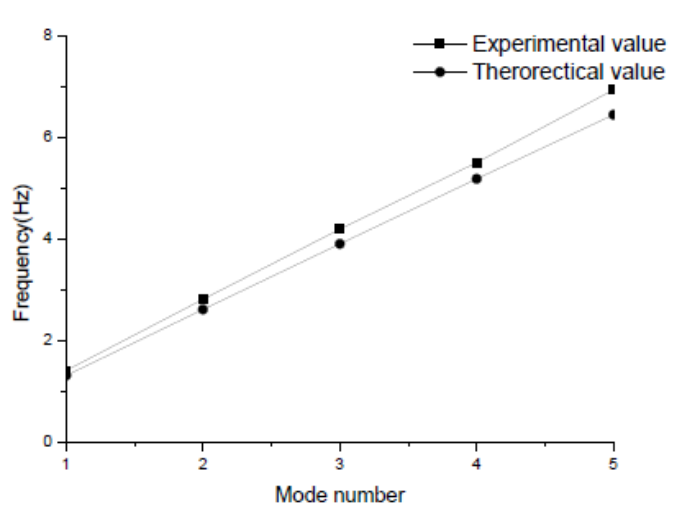

Fig. 7. Comparison of both methods of natural frequency.

From Table II and Fig. 7, it is apparent that experimental value larger than MATLAB value, the percentage of error is $7 \%$ at mode 1 , and error is increased with the order number raise. The difference in the results are due to the fact that the boundary conditions are fixed bearing, which strengthen the boundary condition of conductor, giving rise to the experimental value larger than the MATLAB value. It is also important to note that the natural frequencies of conductor with damper less than those of conductor without. The addition of the damper in the span decreases the natural frequencies of the system. This is not surprising as it is known that natural frequency is inversely proportional to the mass.

\section{Evaluation of the Damper Performance}

At each of these test frequencies the following shall be recorded: a) frequency $f$, b) conductor bending strains, c) the exciting force $F$ (peak value) and conductor accelerated $A$ (peak value) at the point of the application of the force, $\mathrm{d}$ ) the conductor antinode peak to peak amplitude $Y_{j}$, according to the IEEE standard and the conductor were vibrated by electro-dynamic shaker and oscilloscope. The power input $P_{j}$ can be calculated by equation (2).

$$
P_{j}=\frac{1}{4 \pi f} F \cdot A \cdot \sin \theta_{a}
$$

where $\theta_{a}$ is the phase angle between the force and the acceleration, which through adjusted by oscilloscope, reached $\theta_{a}=90^{\circ}$.

For each test frequency the power input $P_{j}$ during the test shall exceed the assumed wind power $P_{w j}$ which shall be calculated from the equation:

$$
P_{w j}=L \times d^{4} \times f^{3} \times f n c\left(Y_{j} / d\right)
$$

where $L$ is the maximum protectable conductor span length for the arrangement under test as agreed between the purchaser and the supplier $(\mathrm{m}), d$ is the conductor diameter (m), $\operatorname{fnc}\left(Y_{j} / d\right)$ is the wind power input function as given in Fig. 8 [4]. The conductor were tested at 20\%RTS, and tested 12 uniform distribution frequencies during $15-40 \mathrm{~Hz}$.

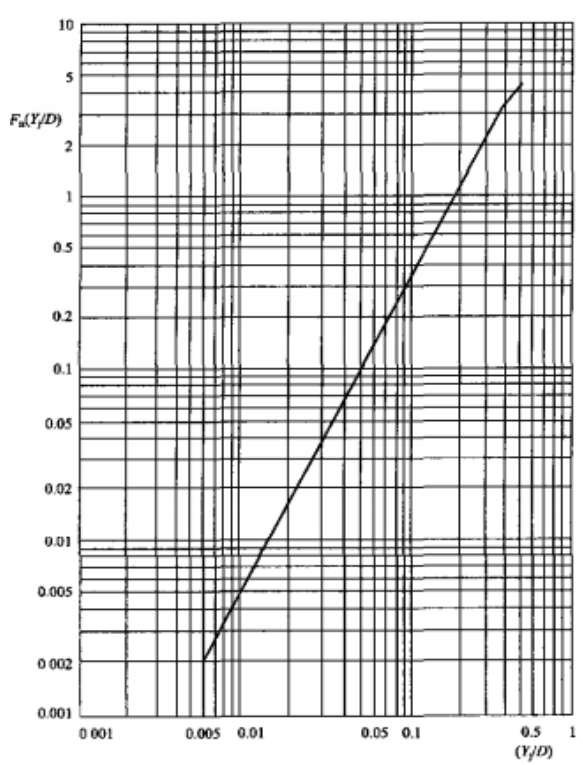

Fig. 8. Wind power input curve.

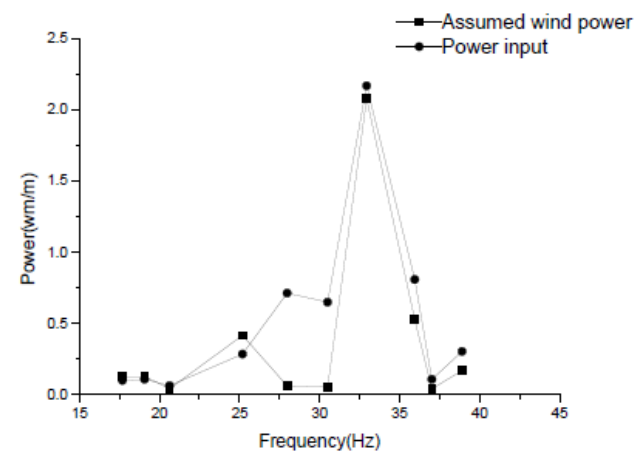

Fig. 9. Comparison of power at RTS=20\%.

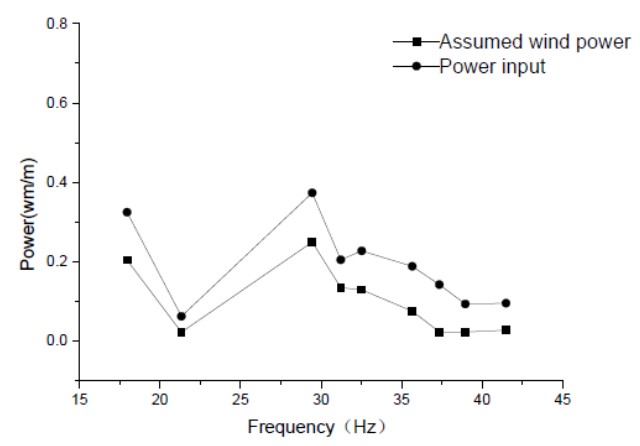

Fig. 10. Comparison of power at RTS=30\%.

The results in Fig. 9 showed that for each test frequency the power input $P_{j}$ during the test exceed the assumed wind power $P_{w j}$ except the frequency $f=25.185 \mathrm{~Hz}$, which maybe an error caused by the operation of oscilloscope. Besides, Fig. 10 shows that for each test frequency the power input $P_{j}$ during the test exceed the assumed wind power $P_{w j}$ strictly. Therefore, it is concluded that the performance of damper meet the demand.

\section{No-dimensional Damping Coefficient $h$}

The no-dimensional damping coefficient, $h$, can be calculated as follow equation (2), by diving the energy introduced in the conductor $E_{\text {diss }}$.by the total kinetic energy 
of the conductor $E_{\text {kin }}$. The energy introduced in the conductor given equation (3)

Being the total kinetic energy of the conductor given by equation (4).[12-14]

$$
\begin{gathered}
h=\frac{E_{\text {diss }}}{4 \pi E_{k i n}} \\
E_{\text {diss }}=\frac{1}{4 \pi f} F A \cdot \sin \theta_{a} \cdot L \\
E_{\text {kin }}=\frac{1}{4} m L w^{2} Y_{j}^{2}
\end{gathered}
$$

where $w$ is circular frequency, other parameters has described above.

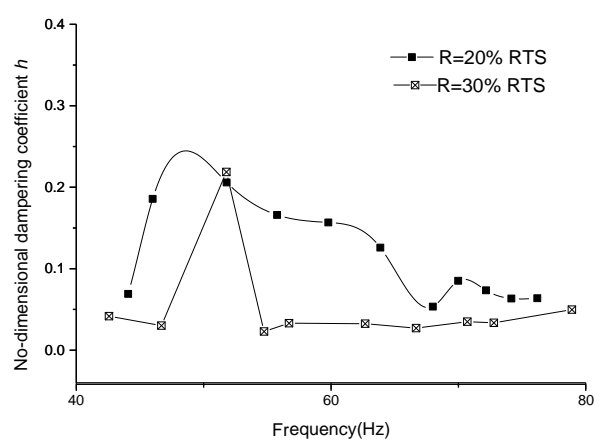

Fig. 11. Comparison of $h$ at RTS $=20 \%$ and RTS $=30 \%$.

Fig. 11 shows that no-dimensional damping coefficients $h, \quad$ at $20 \%$ RTS are higher than those at $30 \%$ RTS. There is not surprising since the self-damper of conductor decreases with the increasing tension.

\section{Conclusions}

The following conclusions are inferred from the analyses.

1) The frequencies can be calculate through three methods, and MATLAB natural frequencies of conductor without damper are close to the theoretical method

2) Experimental natural frequencies are larger than MATLAB natural frequencies, and the frequencies would decrease with the addition of damper.

3) When addition of damper, the increase of the total mass more than the increase of the stiffness.

4) The damper in line with the IEE standard, IEE standard is a good way to verity the damper performance, and further research can be based on IEE standard.

5) When doing anti-vibration design, it should take into full account the no-dimensional damping coefficients at different tension.

\section{ACKNOWLEDGMENT}

The financial support from Guangdong Electric Power Company Research Institute is gratefully acknowledged.

\section{REFERENCES}

[1] Q. Y. Zhen, Aeolian Vibration of Transmission Lines, Beijing: China hydraulic Press, 1987.

[2] C. H. Williamson and R. Govardhan, "Vortex-induced vibrations, annual review of fluid,” Mechanics, vol. 36, pp. 413-555, 2004.

[3] G. Diana and M. Falco, "On the forces transmitted to a vibrating cylinder by a blowing fluid.," Mechanics, vol. 6 , no. 1, pp. 9-22,1971.

[4] Overhead Lines - Requirements and Tests for Stockbridge Type Aeolian Vibration Dampers, IEC Standard 61897, 1998

[5] Overhead Lines - Requirements and Tests for Fittings, IEC Standard 61284,1997, 2nd ed.

[6] IEEE Guide on the Measurement of the Performance of Aeolian vibration Dampers for single Conductors, IEEE Committee, pp. 664-1993

[7] L. Li, Y. K. Chen, Z. X. Xie, and P. Yin, "Indoor simulation tests for the aeolian vibration of overhead lines," Journal of Huazhong University of Science and Technology, vol. 38 , no. 8, pp. 99-102, Aug. 2010.

[8] S. M. Meng and W. Kong, The Design of Transmission Lines, Beijng: China Electric Power Press, 2013, pp. 181-199.

[9] O. Barry, D. C. D. Oguamanam, and D. C. Y. Lin, "Aolian vibration of a single conductor with a stockbridge damper," Journal of Mechanical Engineering, vol. 227, no. 5, pp. 935-945, May 2012.

[10] O. Barry, J. W. Zu, D. C. D. Oguamanam, "Forced vibration of overhead transmission line, analytical and experimental investigation," ASME J. Vib. Control, vol. 136, no. 4, p. 041012, May 2014.

[11] O. Barry, J. W. Zu, and D. C. D. Oguamanam, "Nonlinear dynamics of stockbridge dampers," Journal of Dynamic Systems, Measurement, and Control," vol.137, pp. 0610171-0610177.

[12] R. Claren and G. DIana, "Mathematical analysis of transmission line vibration," IEEE Trans. Power Apparatus Syst , pp. 1741-1771.

[13] H, Wang, J. Miao, J. Luo, F. Huang, and L. Wang, "The free vibration of long-span transmission line conductors with dampers," J. Sound Vibr., vol. 208 , no. 4, pp. 201-516, Dec. 1997.

[14] M. Kraus, and P. Hagedorn, "Aeolian vibration: wind energy input evaluated from measurements on an energized transmission lines," IEEE Trans. Power Delivery, vol. 6, no. 3, pp. 89-106.

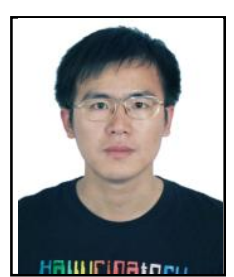

overvoltage analysis.
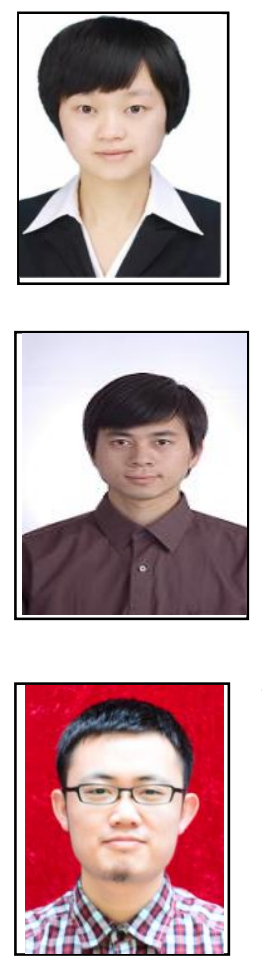

W. P. Xie was born in Jiangxi province, China, in 1986. He received the master degree in engineering mechanics from Huazhong University of Science and Technology, Wuhan, China. in 2012

Currently, he work in Electric Power Research Institute of Guangdong Power Grid Co., Ltd, Guangzhou, 510080, China. His currently research interests include structure fatigue research, structural optimization of power equipment and power system

Tian Peng was born in Jiangxi province, China, in 1992. She is a second-grade postgraduate in civil engineering in Tongji University, Shanghai, China.

Her currently research interests include structure fatigue research, aeolian vibration research of transmission lines, wind resistance of transmission lines.

Kai Xiao was born in Hunan province, China, in 1986. He received the master degree in materials science and engineering from Zhejiang University, Hangzhou, 310000 , China in 2011.

Currently, he work in Electric Power Research Institute of Guangdong Power Grid Co., Ltd, Guangzhou, 510080, China. His currently research interests include structural optimization of power equipment and power system overvoltage analysis and monitoring technology of transmission lines.

Jian Zhang was born in Inner Mongolia, China, in 1992. He is a first-grade doctoral students in civil engineering in Tongji University, Shanghai, China.

His currently research interests include wind resistance of transmission lines, transmission tower strengthening, transmission tower-line system analysis. 